\title{
Development of NC Power Based on Buck Circuit
}

\author{
Fengzhi Dai*, Yuxing Ouyang, Runhua Mao, Ce Bian, Baochang Wei, Yiqiao Qin, Shengbiao Chang, Qijia Kang \\ College of Electronic Information and Automation, Tianjin University of Science and Technology, \\ 1038 Dagunanlu Road, Hexi District, Tianjin, 300222, China \\ E-mail:*daifz@tust.edu.cn \\ www.tust.edu.cn
}

\begin{abstract}
This paper develops a kind of power-supply module that uses digital control and buck circuit. The module can convert $220 \mathrm{~V}$ alternating current into $0-30 \mathrm{~V}$ direct current. In the aspect of circuit design, it is mainly AC-DC, DC-DC conversion circuit and SCM minimum system circuit. In the aspect of control circuit, for feedback signal of current sensor, the power-supply module can obtain average value by multiple methods and remove the mutation value.
\end{abstract}

Keywords: Buck circuit, SCM, AC-DC conversion circuit, DC-DC conversion circuit

\section{Introduction}

With the development of power electronic technology and digital control technology, more devices combine these two technologies. This paper develops a power module that combines MCU with Buck circuit to enhance the digital control of the power supply module to a certain extent to make up for the lack of traditional power supply module ${ }^{1}$.

At present, the traditional power conversion circuit uses TL494 as the PWM signal generator and the MOS tube as the switch element. The regulation of voltage is realized by building analog circuits and adjusting the resistance of sliding rheostat or potentiometer to change the duty ratio of PWM.

A closed-loop system can also be formed by building an analog circuit, but this closed-loop system has a simple structure and low anti-interference ability. The most important thing is that this conversion method that based on the analog circuits has its own insurmountable shortcomings, such as the inability to display parameters of voltage and current in real time and the disadvantage of not being able to be adjusted in real time. Moreover, the conventional power conversion circuit has a complicated structure, low stability, easy to generate errors, and consumes a certain amount of energy because it needs to withstand a large amount of resistance.

The main circuit of the NC power based on Buck circuit is divided into the following three modules: (1) the first module is the AC-DC conversion circuit. Its function is to convert the $220 \mathrm{~V}$ AC to the $30 \mathrm{~V}$ DC through a transformer, a bridge rectifier module and filter capacitor. (2) The second module is the DC-DC conversion circuit. Its function is to convert the $30 \mathrm{~V}$ DC to the $0 \sim 30 \mathrm{~V}$ DC through the Buck circuit. (3) The third module is the microcontroller control circuit, its main function is to protect the special requirements of constant voltage or constant current, through reading the current sensor feedback signal and adjust the PWM duty cycle to change the voltage output ${ }^{2}$.

This paper is organized as follows. Section 2 describes the hardware circuit design, and Section 3 introduces the control strategies. The main circuit simulation is implemented in Section 4, and the conclusion is presented in Section 5. 


\section{The hardware circuit design}

The basic circuit of the system is divided into step-down rectifier filter circuit, Buck circuit, measurement circuit, SCM supply circuit, 430F149 minimum system circuit, LCD liquid crystal display circuit and independent key circuit. The step-down rectifier filter circuit is discussed in Section 2-1. Section 2-2 describes the Buck circuit. Section 2-3 introduces the 430F149 minimum system circuit ${ }^{3}$.

\section{2-1. The step-down rectifier filter circuit}

This part includes the transformer, full-bridge rectifier and filter capacitor. The role of the transformer is to convert the $220 \mathrm{~V}$ AC to $30 \mathrm{~V}$ AC. After the rectification action of the full bridge rectifier module, the alternating current is converted to direct current, but the waveform of the direct current is unstable. Using the filter capacitor can make the DC waveform more stable ${ }^{4}$. The step-down rectifier filter circuit is shown in Fig.1.

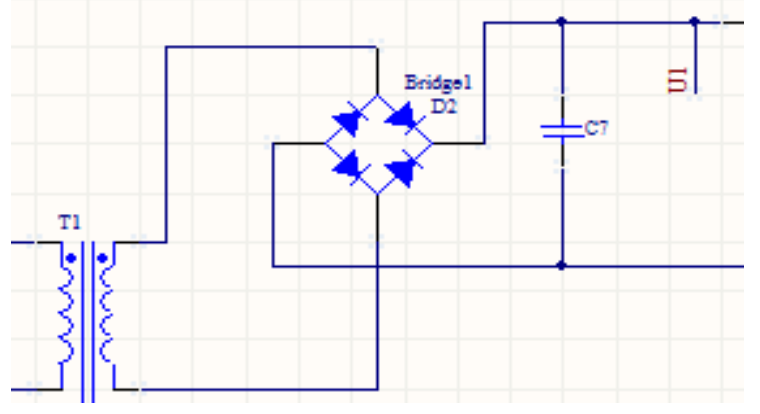

Fig.1 The step-down rectifier filter circuit

\section{2-2. The Buck circuit}

Buck circuit is mainly composed of the MOS tube IRF540, inductor, capacitor and freewheeling diode. The MOS tube IRF540 is mainly used in the circuit as a high-power switching components, which is the core of the DC-DC circuit. The diode protects the entire circuit by preventing the reverse flow of current. Inductance and capacitance form a low-pass filter, making the circuit output current waveform be smoother. The buck circuit is shown in Fig.2.

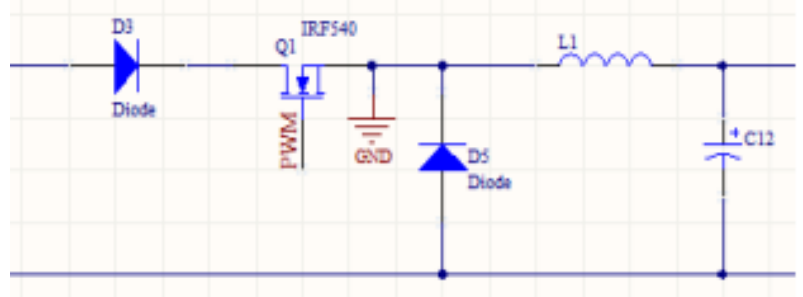

Fig.2 The buck circuit

\section{2-3. The circuit of the system}

Other circuits are related to the control chip. The 430F149 microcontroller peripheral circuits include the reset circuit, crystal oscillator circuit and power supply circuit. The power supply circuit mainly uses the LM7805 regulator module and LM117 regulator module. This part of the circuit can change the $30 \mathrm{~V}$ DC voltage into $3.3 \mathrm{~V}$ DC voltage, so as to provide a stable voltage to control the microcontroller. The circuit of the system is shown in Fig.3.

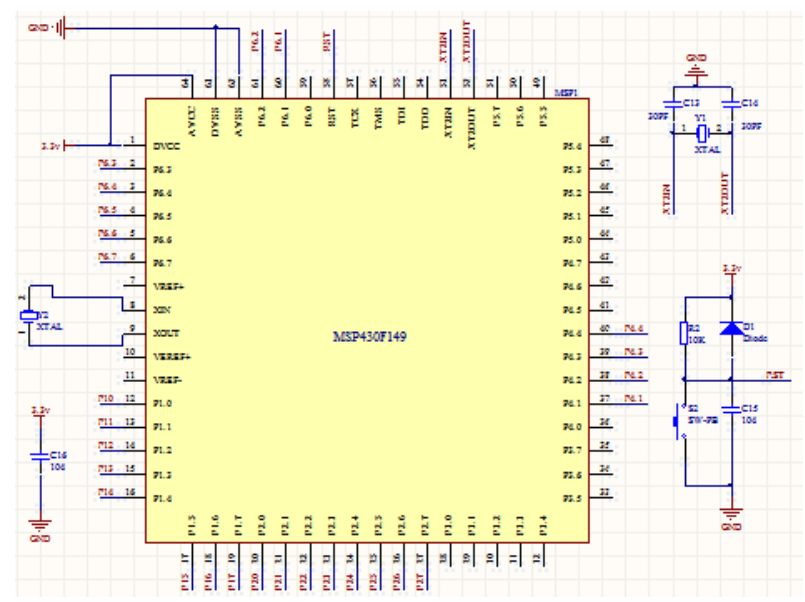

Fig.3 The circuit of the system

\section{The control strategies}

Well-designed hardware circuits and powerful control ICs play a crucial role in NC power, but control strategies are also an integral part to maintain the proper operation of a device ${ }^{5}$. For the NC power that is developed in this paper, the control strategy is mainly used in the chips on the ACS712 current sensor module for the feedback signal processing. The system is based on the signal to adjust the PWM signal output. However, to solve some of the sensor's problems, we can improve 
the situation to some extent through data processing. The PWM signal output must also have a certain control strategy, in order to improve the voltage conversion capability ${ }^{6}$.

Because the ACS712 current sensor feedback is an analog signal, when the external interference has an abrupt change in value, there will be a measurement error. The control strategy adopts the method of filtering the mutation value and obtaining the average value. In each group of data, five data that have larger absolute deviation value are deleted, and the five data that have smaller absolute deviation value are retains. Thus for the five groups, there are a total of 25 sets of data. We again average these 25 data, the average as the true value of the feedback signal.

After obtaining the feedback value of the data processing, we use the difference between the feedback value and the value that has been set to adjust the duty cycle of the PWM. Because the load will have a certain voltage and current limit, so we adjust the PWM duty cycle, the step value should not be too large, to prevent overshoot. However, if the step value is too small, it will make the adjustment time increase, which will not be conducive to real-time response of the entire system. Therefore, we set the threshold to adjust the PWM duty cycle. When the difference between the actual feedback value and the set value is larger, we adopt a larger step value to adjust the duty cycle of the PWM, and when the actual feedback value approaches the set value, the step value is reduced.

Through this strategy, the voltage conversion ability of the entire system can be well improved, and the real-time response of the whole system is good.

\section{The main circuit simulation}

The maximum input voltage of LM78XX series voltage regulation module is $36 \mathrm{~V}$, so the voltage after the filter module of variable voltage rectifier must be reduced below 36V. In the Buck circuit, the parameters of capacitance and inductance will directly affect the time of the system circuit to reach the steady-state. So, we need to go through a software simulation to find one or more sets of optimization data. Section 4-1 introduces the AC-DC conversion simulation circuit. Section 4-2 introduces the buck circuit simulation.

\section{4-1 The AC-DC conversion simulation circuit}

In the AC-DC conversion simulation circuit, we set three measurement points, namely the input voltage $\mathrm{Ui}$, the transformer output voltage U1i and the rectified output voltage U1. We use voltmeters and oscilloscopes as measurement tools. The oscilloscope detects the waveform of the final output voltage $\mathrm{U} 2$ and the time it takes for the voltage to reach to the steady-state time. The simulation result of AC-DC circuit is shown in Tab.1.

Tab.1 The simulation result of AC-DC circuit

\begin{tabular}{|c|c|c|c|c|c|c|}
\hline Ui (V) & n1:n2 & U1i (V) & U1 (V) & C (uF) & T(ms) & W \\
\hline 220 & $7: 1$ & 31.149 & 42.357 & 500 & 4.924 & S \\
\hline 220 & $8: 1$ & 27.492 & 36.768 & 500 & 4.924 & S \\
\hline 220 & $9: 1$ & 24.437 & 32.688 & 500 & 4.924 & S \\
\hline 220 & $10: 1$ & 21.993 & 29.234 & 500 & 4.924 & S \\
\hline
\end{tabular}

(Note: C: Filter capacitor, T: steady-state time, W: Waveform, S: stable.)

If we hope that the final output voltage of the overall circuit $\mathrm{U} 2$ ranges between $0 \sim 30 \mathrm{~V}$, then in the AC-DC circuit, the output voltage values $\mathrm{U} 1$ that is after rectification must be above $30 \mathrm{~V}$. Taking into account the MOS tube IRF540 protection and as much as possible to reduce the heating power, the AC-DC circuit output voltage values should be as close to $30 \mathrm{~V}$. According to the above two points, when we select the transformer turns ratio is 9: 1 turns, the circuit can make the output voltage value $\mathrm{U} 1$ is $32.688 \mathrm{~V}$.

\section{4-2 The buck circuit simulation}

Buck circuit is the core circuit of the NC power supply, and it will directly affect the efficiency of the entire system, so the parameters of each component is particularly important ${ }^{7}$. All the parameters of the capacitance and inductance in the Buck circuit are determined after simulation, so as to keep the output waveform stable, reducing the time of the Buck circuit to reach the steady state ${ }^{8}$.

From the experimental data in Tab.2 we know that the values from simulation and theoretical calculation are different. Experimental data show that when we select the capacitance and inductance larger values, the longer the time is required to reach the steady state, but the waveform is relatively smooth. When the selected 
capacitance and inductance is small, although the steady-state time is shorter, the waveform is unstable. So we choose the middle value of the capacitance and inductance as $470 \mathrm{uF}$ and $100 \mathrm{mH}$.

Tab. 2 The simulation result of Buck circuit

\begin{tabular}{|c|c|c|c|c|c|c|}
\hline $\mathrm{U} 1(\mathrm{~V})$ & $\mathrm{D}$ & $\mathrm{TV}(\mathrm{V})$ & $\mathrm{SV}(\mathrm{V})$ & $\mathrm{L}_{1}(\mathrm{mH})$ & $\mathrm{C}_{12}(\mathrm{uF})$ & $\mathrm{S}(\mathrm{ms})$ \\
\hline 32.688 & $20 \%$ & 6.538 & 5.915 & 100 & 470 & 203.4 \\
\hline 32.688 & $40 \%$ & 13.075 & 12.590 & 100 & 470 & 247.6 \\
\hline 32.688 & $60 \%$ & 19.613 & 19.388 & 100 & 470 & 255.2 \\
\hline 32.688 & $80 \%$ & 26.240 & 25.981 & 100 & 470 & 272.1 \\
\hline 32.688 & $90 \%$ & 29.412 & 29.285 & 100 & 470 & 250.5 \\
\hline 32.688 & $90 \%$ & 29.412 & 29.149 & 1000 & 470 & 397.4 \\
\hline 32.688 & $90 \%$ & 29.412 & 29.275 & 10 & 470 & 101.7 \\
\hline 32.688 & $90 \%$ & 29.412 & 29.275 & 100 & 47 & 68.7 \\
\hline 32.688 & $90 \%$ & 29.412 & 29.338 & 100 & 4700 & 799.9 \\
\hline
\end{tabular}

(Note: D: Duty cycle, TV: Output theoretical value, SV:

Output simulation value, S: Steady-state time)

From the circuit simulation results, we determine the transformer turns ratio of 9:1, and in the Buck circuit, the specific value of the capacitor is 470uf and the inductor value is $100 \mathrm{mH}$.

\section{Conclusion}

Developed in this paper based on the Buck circuit NC power supply, it can output any stable voltage values between $0 \sim 30 \mathrm{~V}$, and the values of which can be real-time displayed. The device can not only be used as a constant current source, but also can be freely switched to a constant voltage source.

The working hours of the equipment can be set programmatically, and the equipment can automatically calculate and display the voltage, current and consumed energy values through the load. It has the automatic alarm function that is used to monitor these values to reach the limited value or not. At the same time, we incorporated data processing and control strategies to ensure the stability of the equipment and formed a closed-loop control. In the whole system, the analog circuit part is responsible for the voltage conversion, while the digital control part is responsible for the regulation of the system. These two parts work independently and complement each other, so that the power supply module is smart and the device is safer and steadier to supply the power to the load.

\section{Acknowledgements}

The research is partly supported by the Research Fund for the Reform in Education from Tianjin Municipal Education Commission of China (171005704B).

\section{References}

1. Chun-Wei Feng, Wei-Feng Min, Juan Lei. Design of a High-precision Numerical Control LED Constant Current Driver. Electronics World, 14(14): 113-114, 2017.

2. Yin-qiao Li, Yu-Ling Liu, Xue-Feng Liu, Shan Huang. Design of NC DC power supply based on MSP430. Computer Measurement and Control, 24(12): 231-233+236, 2016.

3. Zhen-Feng Xiao, Rong-Xiang Yuan, Xiang-Tian Deng, Xiao-Lei Liu. Remote Intelligent Fault Monitor Based on MSP430F169. Electric Power Automation Equipment, 33(01): 160-164, 2013.

4. Zheng-Sen Jiang. Homemade Raiders of Precision CNC Power Supply. Journal of Radio, (9): 58-63, 2012.

5. Xiu-Hua Wang. NC Constant Current Source Based on Integrated Switching Regulator Chip. Power Technology, 5: 738-739, 2012

6. Tian-Chen Huang, Song Jia, Jian-Hua Yu. Design and Realization of High Precision NC DC Current Source. Instrumentation Technology and Sensor, (6): 27-29, 2013.

7. Zhong-Hua Yun, Huan-huan Yin, Yong-Feng Li. Simulation and Research of DC Buck Chopper in Multisim. Application Engineering of Computer Engineering, (16): 216-218, 2015.

8. Zhao-Lin Huang, Wen-Tong Wu, Xiao-Jun Jing, et al Design of precision numerical control direct current power supply. Control Automation, 8(8): 134-137, 2011. 